\title{
Princípios para análise do uso de sistemas de informação: proposta baseada nos estudos de redes conceituais
}

\author{
Arnaldo Alves Ferreira Júnior \\ Doutor; Universidade Federal de Goiás, Goiânia, GO, Brasil; \\ arnaldojunior@ufg.br \\ Marcelo dos Santos \\ Doutor; Universidade de São Paulo, São Paulo, SP, Brasil; \\ mar.santos@usp.br
}

\begin{abstract}
Resumo: Há anos, os estudos e avaliações de uso de produtos e/ou serviços disponibilizados em ambientes digitais ganharam importância, objetivando a produção de soluções que ofereçam facilidade de uso e experiências agradáveis aos seus usuários. Ocorre, entretanto, que os usuários compreendem o mundo de maneira diferente e, consequentemente, suas necessidades de informação também são diferentes. Assim, partindo dessa premissa, o principal objetivo desta pesquisa foi buscar a identificação e caracterização de princípios norteadores para análise do uso de sistemas de informação em ambientes digitais. A pesquisa foi conduzida com base nos estudos de redes de relacionamentos conceituais. Para tanto, utilizou-se uma infraestrutura (framework) conceitual e, a partir das formas e comportamentos apresentados pelas conexões conceituais, foram observados e extraídos princípios para análise de uso dos sistemas de informação. Trata-se, portanto, de uma pesquisa qualitativa, baseada no Método Bibliográfico e Levantamento (survey) em fóruns eletrônicos de discussão e, de modo complementar às análises, foram utilizados métodos, técnicas e medidas dos estudos de redes para identificação e caracterização dos princípios para avaliação de sistemas de informação. Como resultado, destaca-se que os princípios identificados representam elementos norteadores para a composição de parâmetros e criação de procedimentos que auxiliam na análise dos usos de sistemas de informação em geral. Não se trata de substituição dos métodos e técnicas amplamente utilizados nas áreas de Interação Humano Computador (IHC), Usabilidade, Análise de Sistemas e/ou Engenharia de Requisitos; contudo, os referidos princípios representam contribuições ao estudo das interações entre pessoas e sistemas de informação, considerando-se os relacionamentos entre os conceitos que essas pessoas ativam e/ou mobilizam para interpretar esses contextos de uso.
\end{abstract}

Palavras-chave: Uso de informação. Comportamentos de busca e uso de informação. Sistemas de informação. Interação Humano Computador. Estudos de redes. 


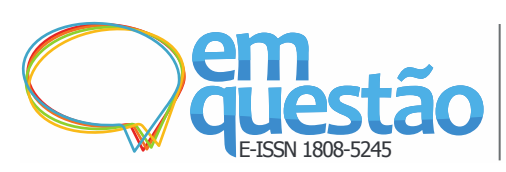

\section{Introdução}

Os estudos e avaliações de uso de sistemas de informação oferecidos em ambientes digitais passaram a ter maior importância com a abertura da internet ao setor comercial, no início da década de 1990 (CASTELLS, 2003), quando milhares de páginas eletrônicas e conteúdos digitais precisaram apresentar funcionalidades e facilidades de uso para viabilizar o acesso a diversos grupos de usuários. Assim, a facilidade de uso e a oferta de experiências agradáveis também passaram a ser objetivo de profissionais que trabalham na implementação e oferta de serviços mediados pelas Tecnologias da Informação e Comunicação (TICs) (BEDERSON; SCHNEIDERMAN, 2003).

Para tanto, compreendeu-se que os investimentos em padrões de qualidade, em relação ao uso de sistemas de informação, são fatores que podem melhorar a sua utilização, considerando-se as diferentes demandas e o uso desses sistemas no cotidiano (SANTA ROSA; MORAES, 2008). Isso tem como pressuposto que, ao facilitar o uso de um sistema de informação, como consequência, o aprendizado será mais rápido e eliminar-se-á barreiras no uso, como aquelas que dificultam e/ou impedem o acesso a esses sistemas pelos diversos usuários (reais e/ou potenciais).

Particularmente, nas interações do usuário com os sistemas de informação, percebe-se que o comportamento informacional - relacionado à busca, uso e manejo de informações e fontes para satisfazer as necessidades informacionais - é um dos pilares que sustentam a relação que os usuários estabelecem com os sistemas de informação. Assim, no que diz respeito ao uso desses sistemas, perpassa a ideia de que existe uma rede de conceitos relacionados aos comportamentos de busca, uso e compreensão da informação. Tal ideia viabiliza investigar as variáveis envolvidas nesses processos, com base na formalização dos relacionamentos entre os conceitos envolvidos, utilizandose estruturas de rede e a visualização na forma de grafos. Os conceitos analisados nesta pesquisa, na forma de redes, têm como base o fato de que grupos de indivíduos compreendem o mundo de maneira diferente e, consequentemente, suas necessidades de informação também são diferentes. 
Entende-se que os indivíduos, com base em experiências cotidianas, adquirem conceitos e, internamente, organizam-nos e relacionam-nos com outros conceitos, mimetizando redes de relacionamentos conceituais. Nesse caso, qualquer alteração nessas redes, as quais representam compreensões dos indivíduos sobre a realidade, tende a modificar os comportamentos que envolvem a busca, interação, compreensão e uso de informação.

Nesse sentido, o estudo dessas redes representa uma possibilidade de investigar e compreender a maneira através da qual os usuários se relacionam com os sistemas de informação, em particular aqueles sistemas baseados em TIC's. Assim, o referido estudo depende da disponibilidade de uma infraestrutura (framework) conceitual e é a partir das formas e comportamentos apresentados pelas conexões conceituais que se observa e extrai princípios para análise de uso dos sistemas de informação.

Neste trabalho, princípios são compreendidos como elementos norteadores para se analisar o uso dos sistemas de informação, abrangendo os requisitos funcionais e não funcionais destes sistemas. A avaliação destes últimos, devido às suas características, necessita de parâmetros que possam orientá-la. Para tanto, conjectura-se que estes parâmetros contribuirão para com a área de estudos e análises da interação entre usuários e sistemas de informação, uma vez que tais parâmetros poderão servir de base para a elaboração de instrumentos utilizados na avaliação dos usos de sistemas de informação.

O objeto de estudo são os relacionamentos conceituais em rede. Tratase de um constructo teórico pensado a partir de duas situações encontradas na literatura de Ciência da Informação. A primeira está ligada ao argumento de que a transformação do conhecimento acontece pelo confronto de informação nova com estoques de informação já apreendidos pelo indivíduo em suas estruturas mentais. Nesse confronto, as estruturas mentais do indivíduo são alteradas, incorporando informação nova e ampliando as possibilidades de novos raciocínios. A segunda situação é aquela em que se afirma a necessidade de domínio conceitual por parte do indivíduo, para que esse possa partilhar um universo discursivo comum. Nessa situação, a aquisição de conceitos por parte 
do indivíduo amplia seu nível linguístico e, consequentemente, sua capacidade de compreensão.

De início, pode-se imaginar que uma rede de relacionamentos conceituais se assemelha a um mapa conceitual. Entretanto, as características que podemos elencar a respeito de um mapa conceitual são as seguintes: (1) um mapa conceitual é estático, pois configura-se como uma representação imagética e hierarquizada de relações fixas entre conceitos; (2) apresenta um número limitado de relações possíveis entre os conceitos, sendo que a sua natureza fundamental é a relação de gênero x espécie, e realizam apenas a descrição de domínios específicos de conhecimento (CORREIA; SILVA; ROMANO JÚNIOR, 2010).

Diferentemente de mapas conceituais, as redes de relacionamentos conceituais são dinâmicas, o que indica não haver hierarquia conceitual, pois esses relacionamentos são construídos pelas ações e construção de significado atribuído pelos indivíduos. Nessas redes, há um número ilimitado de relações possíveis entre os conceitos, as quais suportam a interpretação do indivíduo em cada situação devido à multidimensionalidade e multiconexidade entre os nós conceituais. Em tais redes, não há ordem de ativação de nós nem limites para a quantidade de nós ativados. Isso se deve ao fato de que a quantidade de nós ativados indica a complexidade e a dimensão da compreensão dos indivíduos naquela situação específica (CORREIA; SILVA; ROMANO JÚNIOR, 2010).

A questão colocada como elemento de investigação situa-se na intersecção das seguintes áreas de estudos: Comportamentos de Busca e Uso da Informação, Interação Humano Computador e sistemas de informação. De modo particular, nessa intersecção, destacam-se os elementos presentes na concepção de sistemas de informação baseados em TICs, objeto empírico deste trabalho. De antemão, sabe-se que os referidos elementos não atuam de forma isolada. Pressupõe-se que a falta de compreensão das relações entre eles dificulta a criação/adoção de estratégias para avaliar o uso de tais sistemas, tendo em vista que tais estratégias dependem de princípios norteadores.

Assim, considerando a necessidade de parâmetros para a preparação de instrumentos de avaliação destinados à análise do uso de sistemas de informação em ambientes digitais, que princípios podem nortear a composição desses 
parâmetros e gerar um corpus de procedimentos, baseados em relacionamentos entre conceitos, para análise do uso desses sistemas? Colocado dessa forma, o problema de pesquisa está relacionado com a elaboração de procedimentos visando à análise do uso de sistemas de informação. Nesse cenário, esta pesquisa estrutura-se a partir das necessidades de se realizar estudos do uso de sistemas de informação, considerando-se os contextos sociais e adoção de tecnologias que auxiliam as pessoas nas mais diversas tarefas do cotidiano. Assim, a investigação das dinâmicas que envolvem o uso de tais tecnologias mostra-se necessária, dado o crescente uso dessas tecnologias em diversos segmentos sociais. Portanto, investigar o uso de sistemas de informação inclui a compreensão dos usos feitos com essas tecnologias, para se compreender os impactos causados por elas e como as pessoas se apropriam e reelaboram tais tecnologias a partir de seus usos.

A partir das premissas acima elencadas, objetivou-se preparar uma infraestrutura (framework) para viabilizar a identificação e caracterização de princípios que possam ser aplicados na análise do uso de sistemas de informação em ambientes digitais. Isso é feito a partir da identificação de critérios e medidas, incluindo desde abordagens utilizadas em estudos das áreas de comportamentos de busca e uso da informação, interação humano computador, sistemas de informação e análise de redes. Especificamente, tal objetivo consistiu em desenvolver princípios de uso da rede de relacionamentos para analisar o uso de sistemas de informação. Como resultado, obteve-se um conjunto de princípios e procedimentos que poderão ser utilizados na construção de instrumentos de análise.

\section{Procedimentos de pesquisa}

Os procedimentos adotados neste trabalho estão organizados, de maneira resumida, da seguinte forma:

a) revisão de literatura sobre a análise de redes, com o objetivo de levantar as técnicas e medidas normalmente utilizadas; 


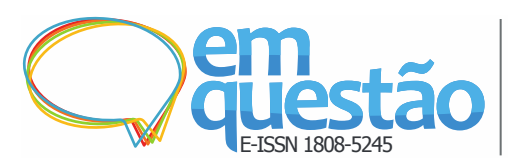

Princípios para análise do uso de sistemas de informação: proposta baseada nos estudos de redes conceituais

Arnaldo Alves Ferreira Junior e Marcelo dos Santos

b) análise e avaliação de quais técnicas e medidas encontradas poderiam contribuir para a análise de sistemas de informação, com base na formalização de redes complexas de conceitos;

c) seleção de técnicas e medidas mais apropriadas ao uso na avaliação de sistemas de informação e, mais especificamente, quais poderão contribuir para construções de modelos conceituais de interação para sistemas de informação, considerando-se as comunidades de usuários atendidas por tais sistemas;

d) seleção de um conjunto de princípios para análise de sistemas de informação com o uso de medidas, a partir dos campos de comportamentos de busca e uso da informação, interação humano computador, sistemas de informação. Cada princípio identificado foi descrito em termos de seus âmbitos de compreensão. A descrição desses componentes não se limitou a identificar cada um deles, mas a descrever a função de cada um, a natureza de existência e o encadeamento deles dentro do contexto de atividade proposto pela pesquisa em questão.

O resultado objetivado foi um conjunto de princípios que poderão nortear as análises que objetivem, dentre outras possibilidades, avaliar qualitativamente o uso de sistemas de informação, a partir dos relacionamentos conceituais que os usuários constroem ao utilizar esses sistemas em atividades cotidianas, sejam elas de trabalho ou lazer. Acredita-se que, baseando-se no fato de que as estruturas cognitivas dos indivíduos mudam a partir de novas informações, elas podem ser mapeadas e modeladas com o uso de redes de relacionamentos conceituais. Assim, é possível verificar o crescimento da compreensão de grupos de usuários de determinados sistemas e/ou tecnologias de informação e comunicação, analisando o crescimento e a dinâmica de comportamentos apresentados por redes de conceitos a partir do relacionamento entre eles.

\section{Resultados e discussão}

Os resultados apresentados nesta seção referem-se às análises feitas a partir dos comportamentos identificados nos grafos elaborados para cada uma das áreas de 


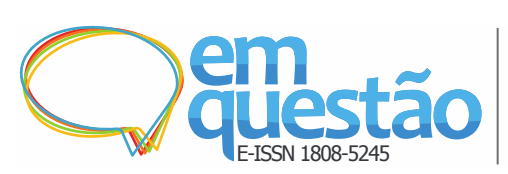

Princípios para análise do uso de sistemas de informação: proposta baseada nos estudos de redes conceituais

Arnaldo Alves Ferreira Junior e Marcelo dos Santos

conhecimento delimitadas na pesquisa. São apresentados os grafos obtidos após a tabulação dos dados levantados e os resultados estatísticos das medidas selecionadas para uso. Cada um dos resultados alcançados nas respectivas medidas foi analisado e discutido, tendo como foco compreender os comportamentos apresentados pela rede conceitual e verificar a presença de algum padrão de regularidade.

A estratégia de utilizar os possíveis padrões regulares de comportamentos identificados na rede mostrou-se uma estratégia viável para verificar a hipótese de pesquisa adotada neste trabalho. A referida hipótese teve como fundamento a possibilidade de uso de relações conceituais como objetos/situações/processos a serem verificados e como sistematizadores de procedimentos na construção de instrumentos de análise, destinados a verificar as diversas variáveis envolvidas no uso de sistemas de informação. Assim, os princípios que se pretende estabelecer, e que, por sua vez, nortearão a análise do uso de sistemas de informação, serão baseados nos comportamentos apresentados por redes de conceitos, representadas e analisadas pelo uso de grafos.

Nas subseções seguintes, são apresentados os resultados alcançados em cada área de conhecimento delineada na pesquisa. A estrutura escolhida para organizar os resultados inicia-se com discussões sobre os comportamentos verificados nos grafos, os resultados estatísticos de cada medida e a análise desses resultados. Em cada uma das subseções são apresentados os respectivos grafos elaborados e as tabelas de dados com os resultados estáticos das redes, separados por medidas referentes aos nós, às arestas que conectam os nós e às medidas globais. Para elaboração dos grafos e das análises estatísticas, foram utilizados os algoritmos de distribuição Fruchterman-Reingold e Force Atlas, bem como o particionamento da rede por Graus dos nós.

\subsection{Princípios de busca, comportamentos, necessidades e usuários de informação}

De maneira geral, pode-se afirmar que mecanismos e estruturas relacionados aos usos que as pessoas fazem de sistemas de informação estão distribuídos ao longo de uma rede e podem ser estudados em suas dinâmicas de micro e macro 
Princípios para análise do uso de sistemas de informação: proposta baseada nos estudos de redes conceituais

Arnaldo Alves Ferreira Junior e Marcelo dos Santos

relacionamentos, tornando possível o mapeamento e a compreensão de diversas variáveis que podem influenciar nos comportamentos relacionados ao uso de sistemas de informação.

A figura 1, a seguir, traz o grafo elaborado para o campo de conhecimentos que agrega os temas sobre Busca, Comportamentos, Necessidades e Usuários de Informação. Com base nos dados levantados e nas ferramentas utilizadas para tabulação, organização e distribuição, foi possível representar graficamente a rede complexa na qual são estruturados os temas em questão. A partir da visualização dos temas organizados em um grafo é possível perceber os temas centrais e, a partir deles, criar mecanismos que avaliem as conexões e dinâmicas envolvidas no uso que as pessoas fazem dos sistemas de informação em geral.

Figura 1 - Grafo da rede sobre Busca, Comportamentos, Competências, Necessidades e Usuários de Informação

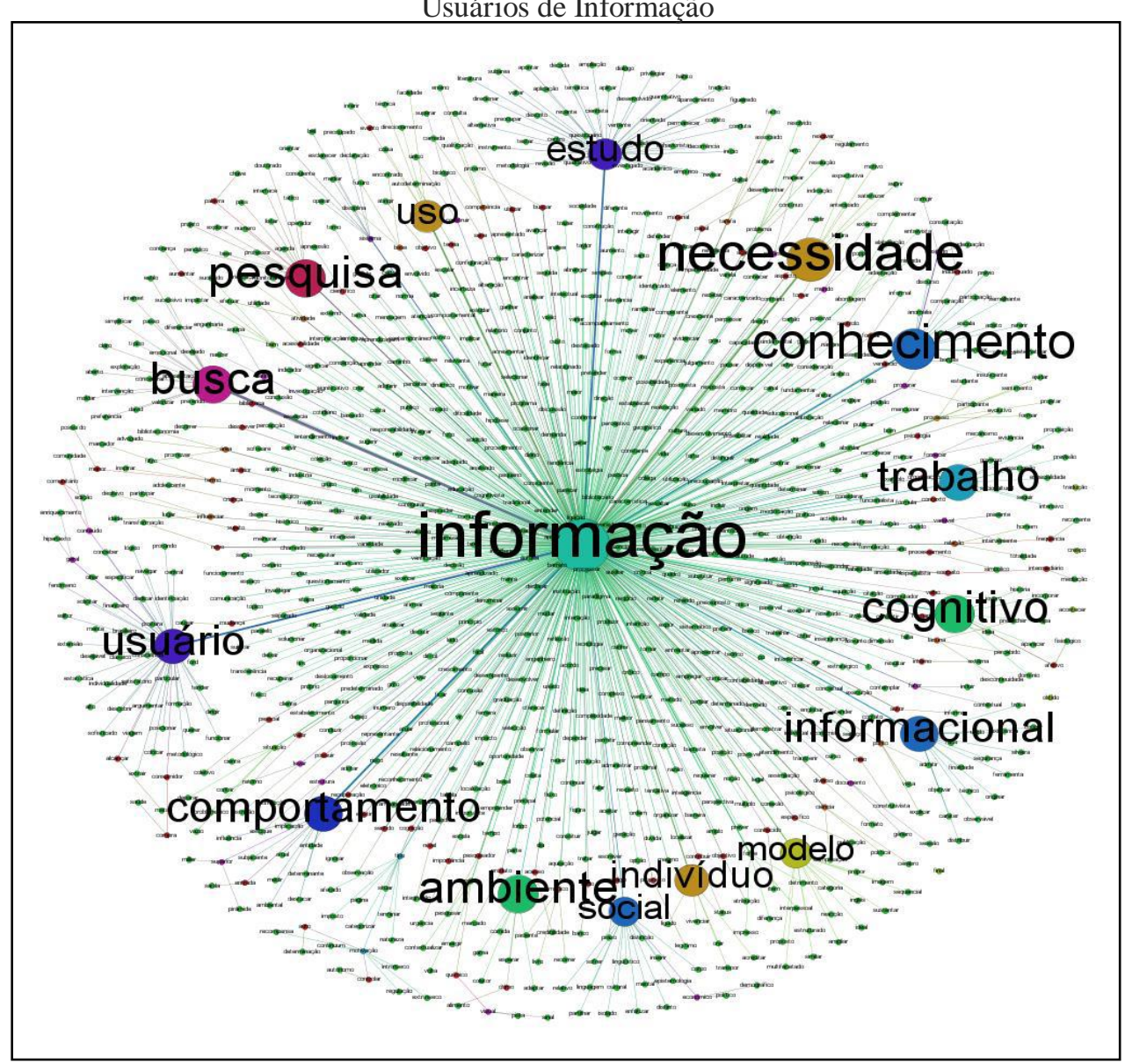

Fonte: Elaborado pelos autores. 
Os resultados encontrados a partir das medidas de redes definidas neste trabalho encontram-se agrupadas na tabela abaixo. Os estudos de redes e o estudo das representações gráficas dessas redes a partir dos grafos contam com uma variedade bastante grande de medidas de análise. A princípio, todos os aspectos de uma rede possuem medidas estatísticas que indicam a situação desses aspectos em relação ao ponto da rede no qual se encontra, em relação a toda rede ou ainda aspectos específicos dos nós da rede, as arestas que ligam os nós, as formas de movimentação de um nó a outro e quais os caminhos mais curtos e mais longos na rede.

Neste trabalho, foram escolhidas cinco medidas para análise da rede sobre Busca, Comportamentos, Necessidades e Usuários de Informação (quadro 1). A escolha de apenas cinco medidas ocorreu devido a um recorte necessário no conjunto de medidas possíveis de serem utilizadas. Outra situação foi a necessidade de abordar os campos de conhecimento com número reduzido de medidas para que cada uma delas pudesse ser verificada em profundidade e testada nos parâmetros iniciais definidos para o trabalho, respeitando as propriedades inerentes aos temas sobre Busca de Informação, Comportamento Informacional, Necessidades de Informação e Usuários de Informação.

Quadro 1 - Medidas da rede sobre Busca, Comportamentos, Competências, Necessidades e Usuários de Informação

\begin{tabular}{|c|c|c|c|}
\hline $\begin{array}{c}\text { Dimensõ } \\
\text { es }\end{array}$ & Medidas & Resultados & \\
\hline \multirow[t]{3}{*}{ Rede } & Grau Médio & 1,988 & $\begin{array}{l}\text { Não há nós totalmente } \\
\text { desconectados. } \\
\text { Estudos e análises que } \\
\text { objetivam investigar sobre } \\
\text { Busca de Informação, } \\
\text { Comportamento } \\
\text { Informacional, Necessidades } \\
\text { de Informação e Usuários de } \\
\text { Informação podem ser feitos } \\
\text { individualmente } \\
\text { combinados entre si. }\end{array}$ \\
\hline & Diâmetro da Rede & 8 & $\begin{array}{l}\text { A transitividade na rede é } \\
\text { relativamente fácil, podendo- } \\
\text { se atingir qualquer ponto com } \\
\text { poucas conexões necessárias. }\end{array}$ \\
\hline & $\begin{array}{c}\text { Densidade do } \\
\text { Grafo }\end{array}$ & 0,002 & $\begin{array}{l}\text { Para que se possa } \\
\text { desenvolver estudos que } \\
\text { abarquem campos como } \\
\text { necessidade de informação e } \\
\text { busca de informação, ou } \\
\text { necessidades de informação e }\end{array}$ \\
\hline
\end{tabular}




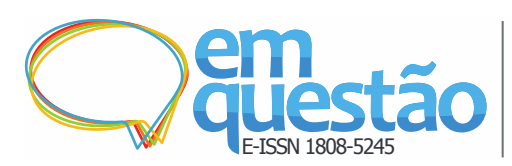

Princípios para análise do uso de sistemas de informação: proposta baseada nos estudos de redes conceituais

Arnaldo Alves Ferreira Junior e Marcelo dos Santos

\begin{tabular}{|c|c|c|c|}
\hline & & & $\begin{array}{l}\text { usuários de informação } \\
\text { específicos, ou ainda as } \\
\text { questões relacionadas ao } \\
\text { comportamento } \\
\text { informacional, é necessário } \\
\text { que alguns nós específicos } \\
\text { sejam considerados no } \\
\text { estudo. }\end{array}$ \\
\hline & $\begin{array}{c}\text { Componentes } \\
\text { Conectados }\end{array}$ & $\begin{array}{c}\text { Componentes fracamente } \\
\text { conectados: } 6 \\
\text { Componentes fortemente } \\
\text { Conectados: } 0\end{array}$ & $\begin{array}{l}\text { Certos nós específicos são } \\
\text { muito importantes na } \\
\text { manutenção da estabilidade } \\
\text { do campo de conhecimento, } \\
\text { são eles que permitem a } \\
\text { transição entre os vários } \\
\text { componentes do grafo e se } \\
\text { apresentam como os pontos } \\
\text { intermediários entre os temas } \\
\text { e problemas do campo de } \\
\text { conhecimento. }\end{array}$ \\
\hline Nós & $\begin{array}{l}\text { Coeficiente de } \\
\text { Agrupamento }\end{array}$ & Número de Comunidades: 69 & $\begin{array}{l}\text { Mecanismos e estruturas } \\
\text { relacionados aos usos que as } \\
\text { pessoas fazem de sistemas de } \\
\text { informação estão distribuídos } \\
\text { ao longo de uma rede e } \\
\text { podem ser estudados em suas } \\
\text { dinâmicas de micro e macro } \\
\text { relacionamentos. }\end{array}$ \\
\hline
\end{tabular}

Fonte: Elaborado pelos autores.

\subsection{Princípios de interação, interface, usabilidade e mediação da informação}

O efeito desse resultado para a análise do uso de sistemas de informação faz-se presente nos momentos em que for necessário acionar um grupo específico de nós com o objetivo de investigar uma dada situação. Pois, ao considerar o mapeamento dos caminhos da rede necessários à interpretação do fenômeno investigado, os nós responsáveis pela conexão dos componentes que compõem o grafo deverão fazer parte desses caminhos. A posição ocupada por esses nós na ordem de importância que pode ser atribuída a eles equivale à mesma dos nós centrais do grafo, pelo fato de que esses nós respondem pela integridade, robustez e pela capacidade de resistência da rede à retirada de nós aleatoriamente.

A figura 2, a seguir, traz o grafo representativo da rede sobre Interação, Interface, Usabilidade e Mediação da Informação. Como pode ser observado, os temas são bastante diversificados e bem distribuídos, formando subgrafos a partir de nós específicos, e há conexões entre os nós mais centrais da rede. O 


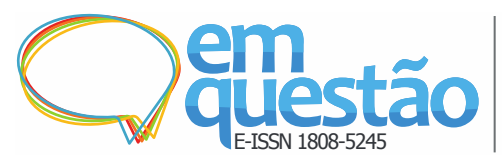

Princípios para análise do uso de sistemas de informação: proposta baseada nos estudos de redes conceituais

Arnaldo Alves Ferreira Junior e Marcelo dos Santos

destaque para essa rede é a ausência de um único nó que concentrasse a distribuição de todo fluxo de informação e respondesse pela manutenção da integridade da rede. A análise visual do grafo também indica os caminhos necessários para conectar os temas principais, sendo possível perceber as ligações estabelecidas entre os nós mais conectados da rede.

Figura 2 - Grafo da rede sobre Interação, Interface, Usabilidade e Mediação da Informação

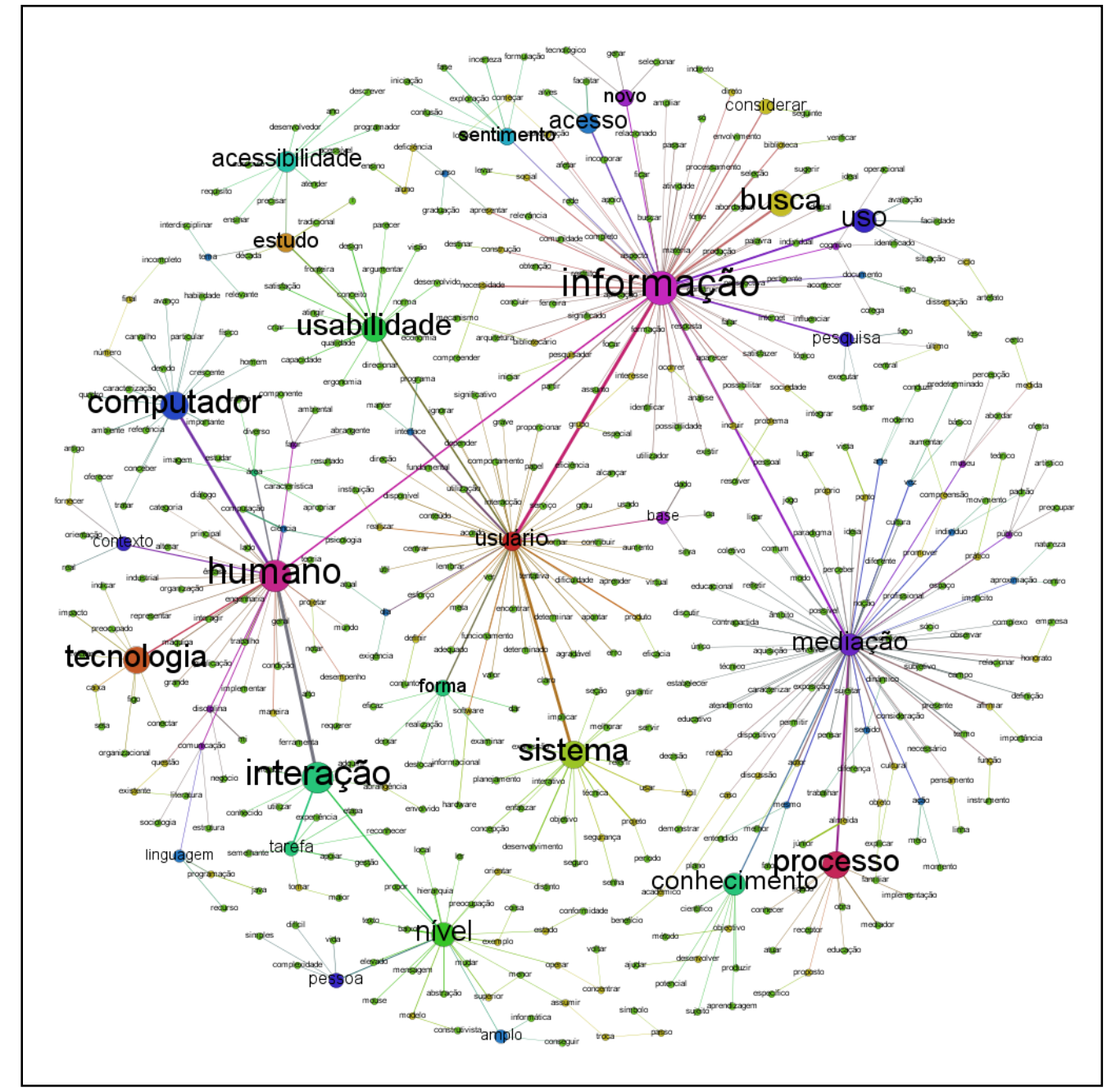

Fonte: Elaborado pelos autores.

O quadro 2, a seguir, traz os resultados obtidos ao se elaborar o grafo para a rede sobre Interação, Interface, Usabilidade e Mediação da Informação. Ao se analisar os resultados reunidos e os grafos representativos da rede desse campo temático, é possível conhecer a dinâmica de organização do campo sob o ponto de vista da análise de redes. Com essas análises, é possível concluir que o campo temático no qual se insere a interação entre usuários e sistemas de informação em geral, as interfaces construídas para a comunicação entre eles, as 
avaliações de usabilidade das interfaces dos sistemas de informação e a mediação que possa haver entre a dimensão humana e a dimensão da máquina ainda precisa de desenvolvimentos que possibilitem a expansão das conexões entre si. Apesar do alto número de temas representados pelos nós da rede, há um baixo número de conexões entre eles. Esses fatos indicam que o relacionamento de temas para o campo precisa ser construído e que os princípios fundamentais que orientam as análises relativas aos problemas dessa natureza temática precisam ser estabelecidos através de investigações e pesquisas que objetivem avaliar situações dos pontos de vista mais diversos dentro campo temático.

Quadro 2 - Medidas da rede sobre Interação, Interfaces, Usabilidade e Mediação da Informação

\begin{tabular}{|c|c|c|c|}
\hline Dimensões & Medidas & Resultados & \\
\hline \multirow{4}{*}{ Rede } & $\begin{array}{l}\text { Distribuição de } \\
\text { Grau } \\
\text { (Conectividade) }\end{array}$ & 1,996 & $\begin{array}{l}\text { É possível integrar em uma } \\
\text { investigação, } \\
\text { multidisciplinares que abordem } \\
\text { simultaneamente processos de } \\
\text { interação, diversidade de } \\
\text { interfaces de sistemas, a } \\
\text { facilidade de uso e os níveis de } \\
\text { mediação da informação } \\
\text { requeridos para uso desses } \\
\text { sistemas. }\end{array}$ \\
\hline & Diâmetro da Rede & 12 & $\begin{array}{l}\text { Maior distanciamento entre os } \\
\text { nós de toda a rede, sendo } \\
\text { necessário o acionamento de } \\
\text { maior número de nós para cobrir } \\
\text { devidamente os fenômenos que se } \\
\text { queira mapear. A possibilidade de } \\
\text { combinação de temas para } \\
\text { investigação é grande. }\end{array}$ \\
\hline & $\begin{array}{l}\text { Densidade do } \\
\text { Grafo }\end{array}$ & 0,004 & $\begin{array}{l}\text { Densidade desse campo é baixa. } \\
\text { Esse fato informa que é } \\
\text { necessário construir as ligações } \\
\text { necessárias ao aumento da } \\
\text { densidade do grafo representativo } \\
\text { da área de Interação, Interface, } \\
\text { Usabilidade e Mediação da } \\
\text { Informação. }\end{array}$ \\
\hline & $\begin{array}{l}\text { Componentes } \\
\text { Conectados }\end{array}$ & $\begin{array}{l}\text { Componentes } \\
\text { fracamente } \\
\text { conectados: } 1 \\
\text { Componentes } \\
\text { fortemente } \\
\text { Conectados: } 0\end{array}$ & $\begin{array}{l}\text { Ao considerar o mapeamento dos } \\
\text { caminhos da rede necessários à } \\
\text { interpretação do fenômeno } \\
\text { investigado, os nós responsáveis } \\
\text { pela conexão dos componentes } \\
\text { que compõem o grafo deverão } \\
\text { fazer parte desses caminhos. }\end{array}$ \\
\hline Nós & $\begin{array}{l}\text { Coeficiente de } \\
\text { Agrupamento }\end{array}$ & $\begin{array}{c}\text { Número de } \\
\text { Comunidades: } 34\end{array}$ & $\begin{array}{l}\text { Com a medida de coeficiente de } \\
\text { agrupamento pode-se, ainda, } \\
\text { analisar a formação de } \\
\text { comunidades de termos em níveis } \\
\text { globais ou locais em toda a rede. }\end{array}$ \\
\hline
\end{tabular}




\subsection{Princípios de tecnologias, sistemas, avaliação e recuperação da informação}

De forma semelhante aos outros grafos representativos das áreas temática dos itens anteriores, sobre essa também é permitido afirmar que a conectividade é facilitada, pois é possível mapear a rede utilizando o grau de conectividade que cada um dos nós apresenta e realizar análise de mobilidade entre eles, identificando regras de movimentação. Esse resultado traz para a análise de uso de sistemas de informação a possibilidade de investigar os temas de forma integrada, conectando os nós necessários à interpretação dos fenômenos que envolvem as tecnologias de informação, os sistemas de informação, a avaliação de tecnologias e sistemas de informação e a recuperação da informação utilizando tecnologias e sistemas de informação. Com esse resultado fica caracterizado a possibilidade de uso dessas características da rede para investigar o uso que pessoas fazem dos sistemas de informação.

A transição entre componentes de Tecnologias da Informação e outros componentes como sistemas de informação, Avaliação de Sistemas e de Informações, ou ainda sobre Recuperação da Informação, torna-se dependente desses nós. Assim, tomando como referência os componentes conectados, para verificar e analisar o uso de sistemas de informação, destaca-se a necessidade de considerar os nós que ligam os diversos componentes do grafo, sob o risco de verificar apenas os temas a partir de seus subgrafos isolados. O grafo apresentado na figura 3, a seguir, traz a representação da rede sobre Tecnologias, Sistemas, Avaliação e Recuperação da Informação; nele é possível verificar a diversidade de temas e visualizar a organização dos nós mais centrais da rede. Também é possível verificar a importância de cada um dos temas a partir das ligações que eles mantêm com nós menos conectados. São esses nós, ao redor dos nós principais, que os caracterizam, conferindo qualidades específicas ao nó principal, tornando possível, à medida que são acionados, o mapeamento e a visibilidade das questões e problemas que o campo temático apresenta e que necessitam de resolução a partir de pesquisas e investigações. 
Figura 3 - Grafo da rede sobre Tecnologias, Sistemas, Avaliação e Recuperação da Informação

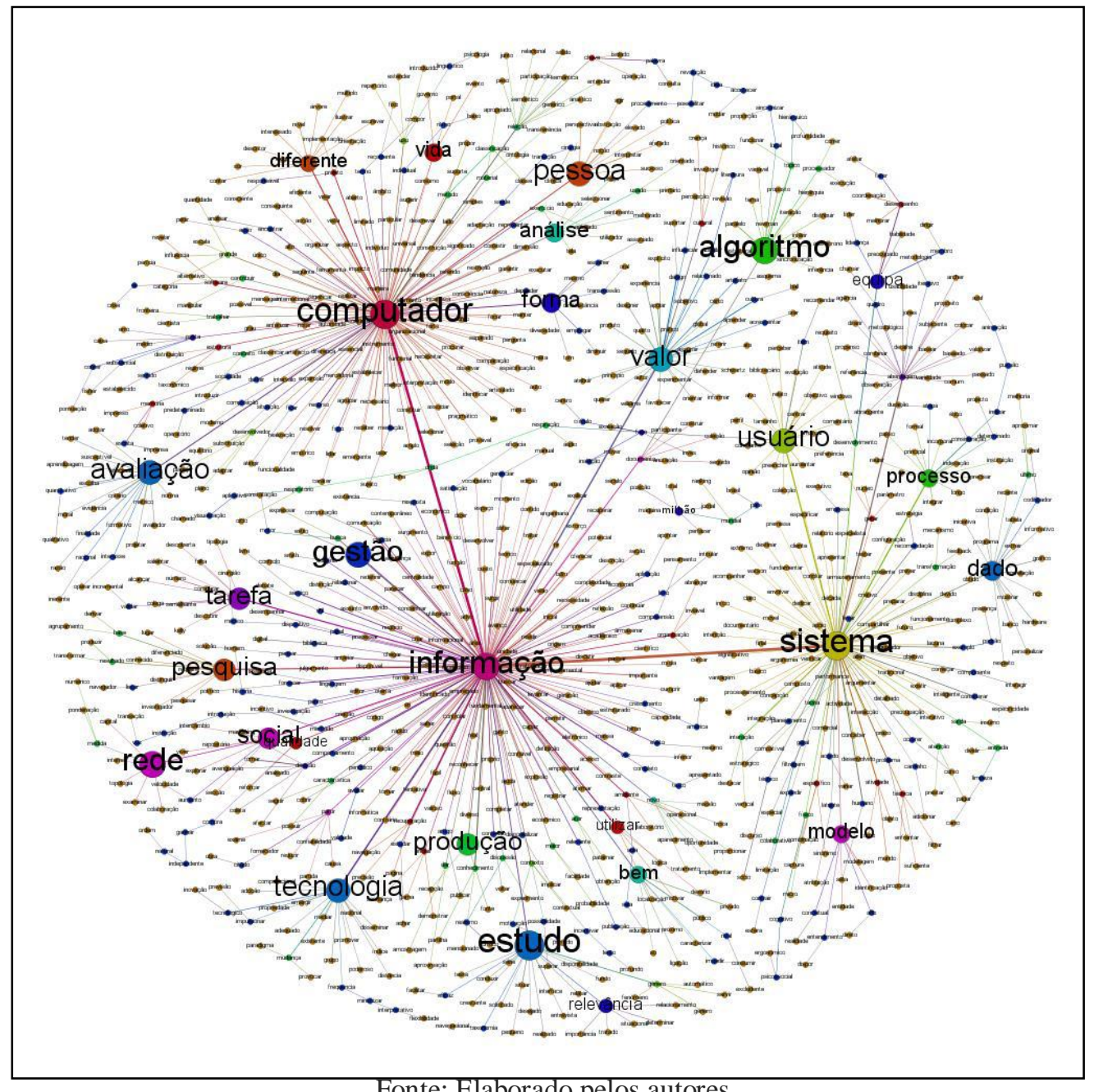

Fonte: Elaborado pelos autores.

O quadro 3, abaixo, traz os resultados das medidas encontradas a partir da análise do grafo sobre Tecnologias, Sistemas, Avaliação e Recuperação da Informação. Com as medidas reunidas, é possível analisar, de maneira integrada, todos os resultados discutidos nos tópicos anteriores e complementar as discussões a respeito dos temas delimitados na pesquisa. A partir da reunião das medidas é possível perceber que a área temática possui uma quantidade relativamente grande de termos, e isso faz com que o diâmetro da rede seja considerado grande se comparado ao diâmetro das outras redes. Outro resultado positivo foi a distribuição de graus, demonstrando a inexistência de nós desconectados. Entretanto, nas medidas de coeficiente de agrupamento, densidade do grafo e componentes conectados, ficou evidenciado a necessidade de maior conectividade entre todos os nós que compõem a rede e o aumento da quantidade de nós que apresentem altos índices de centralidade. 
Esses resultados indicam a precariedade da organização do campo temático e antecipam as dificuldades de se utilizar as dinâmicas da rede como critério de análise do uso de sistemas de informação. Porém, esse fato se apresenta também como oportunidade de desenvolvimento de uma nova abordagem de pesquisa, capaz de trazer resultados ainda não alcançados para o campo temático em estudo.

Quadro 3 - Medidas alcançadas na rede sobre Tecnologias, Sistemas, Avaliação, Recuperação

\begin{tabular}{|c|c|c|c|}
\hline Dimensões & Medidas & Resultados & \\
\hline \multirow{4}{*}{ Rede } & $\begin{array}{l}\text { Distribuição de } \\
\text { Grau } \\
\text { (Conectividade) }\end{array}$ & 1,992 & $\begin{array}{l}\text { É possível mapear a rede utilizando o } \\
\text { grau de conectividade que cada um } \\
\text { dos nós apresenta e realizar uma } \\
\text { análise de mobilidade entre eles, } \\
\text { identificando regras de movimentação. }\end{array}$ \\
\hline & $\begin{array}{l}\text { Diâmetro da } \\
\text { Rede }\end{array}$ & 17 & $\begin{array}{l}\text { A perspectiva de uso dessa } \\
\text { característica para a análise de uso de } \\
\text { sistemas de informação torna-se, } \\
\text { sensivelmente, mais trabalhosa pelo } \\
\text { fato de que mais conexões sejam } \\
\text { necessárias para se mapear os } \\
\text { fenômenos que envolvam tecnologias, } \\
\text { sistemas, avaliação e recuperação da } \\
\text { informação. }\end{array}$ \\
\hline & $\begin{array}{l}\text { Densidade do } \\
\text { Grafo }\end{array}$ & 0,002 & $\begin{array}{l}\text { Possibilidade de fornecer as conexões } \\
\text { necessárias ao aumento da densidade } \\
\text { da rede do campo temático, através da } \\
\text { combinação de temas dentro do } \\
\text { campo com auxílio dos estudos de } \\
\text { redes. }\end{array}$ \\
\hline & $\begin{array}{l}\text { Componentes } \\
\text { Conectados }\end{array}$ & $\begin{array}{l}\text { Componentes } \\
\text { fracamente } \\
\text { conectados: } 4 \\
\text { Componentes } \\
\text { fortemente } \\
\text { Conectados: } 0\end{array}$ & $\begin{array}{l}\text { Entre as possibilidades de se verificar } \\
\text { e analisar o uso de sistemas de } \\
\text { informação na perspectiva dos } \\
\text { componentes conectados deve ser feita } \\
\text { a partir dos nós que ligam os diversos } \\
\text { componentes do grafo }\end{array}$ \\
\hline Nó & $\begin{array}{l}\text { Coeficiente de } \\
\text { Agrupamento }\end{array}$ & $\begin{array}{c}\text { Número de } \\
\text { Comunidades: } 45\end{array}$ & $\begin{array}{l}\text { O coeficiente de agrupamento pode } \\
\text { contribuir para a análise do uso de } \\
\text { sistemas de informação ao identificar } \\
\text { as comunidades temáticas que poderão } \\
\text { ser acionadas para mapeamento dos } \\
\text { fenômenos em estudo. }\end{array}$ \\
\hline
\end{tabular}

Fonte: Elaborado pelos autores.

\subsection{Discussões a partir dos resultados encontrados}

Após a análise das categorias temáticas a partir das medidas de redes e a identificação preliminar de princípios para análise do uso de sistemas de informação, os princípios identificados foram organizados de modo a facilitar a sua compreensão e disposição em um quadro analítico. Em cada área temática, 
foram dispostas as dimensões de rede em análise; especificamente neste trabalho, optou-se por privilegiar a rede e os nós que a compõem. No que se refere à rede, foram selecionadas medidas referentes à Distribuição de Graus (conectividade), Diâmetro da Rede, Densidade do Grafo e Componentes Conectados. Em relação aos nós, foi escolhida a medida que trata do Coeficiente de Agrupamento.

A partir das análises e discussões, em cada resultado medido foi destacado o comportamento específico da rede e dos nós. Esses comportamentos representam as características particulares do campo temático, quando analisado na perspectiva dos estudos de rede. A princípio, pode-se pensar na hipótese de que os princípios encontrados a partir dos resultados de cada medida de rede seriam iguais em todos os grafos elaborados para esta pesquisa. Entretanto, cada princípio foi extraído dos resultados medidos em redes diferentes, com comportamentos e dinâmicas diferentes, e justamente por esse fato os princípios poderão variar, mesmo em situações em que são analisadas as mesmas medidas dos estudos de redes.

Em cada uma das medidas selecionadas para realização desta pesquisa, procurou-se identificar um princípio baseado no comportamento da rede de temas e destacá-lo no escopo da área temática em questão. Assim, para cada resultado alcançado, buscou-se compreendê-lo no contexto das áreas temáticas que originaram esses grafos, e, dessa forma, os princípios identificados devem refletir as dinâmicas de organização e mobilidade das redes às quais pertencem. Nessa perspectiva, esses princípios deverão orientar análises do uso de sistemas de informação para, entre outros objetivos, testar a sua validade para os campos de pesquisa em estudo.

Os princípios encontrados poderão orientar a análise do uso de sistemas de informação a partir da elaboração de grafos representativos de redes complexas que se formam a partir das relações que se estabelecem quando pessoas utilizam tais tecnologias ou sistemas para uma finalidade qualquer. Orientações como a inexistência de nós desconectados, transitividade facilitada pela necessidade de poucas conexões, alta conectividade entre os nós e o agrupamento de temas em redes menores podem contribuir para o crescimento do campo de conhecimento. 
A partir das categorias temáticas vinculadas ao estudo de Comportamentos de Busca e Uso de Informação, pode-se retomar a ideia de que a Busca de Informação é compreendida como a ação desencadeada pelo usuário após a identificação de uma necessidade de informação; de maneira geral, nesse processo, o usuário pode interagir com diversos sistemas de informação de variadas naturezas (MARTÍNEZ-SILVEIRA; ODDONE, 2007). Assim, adotar como princípio a ideia de que a busca de informação acontece de maneira integrada a outros componentes na forma de redes de relacionamentos conceituais nos permite compreender essa categoria de forma reticular, e não necessariamente desencadeada de maneira linear, com processos definidos e iniciados em uma perspectiva protocolar. Tal compreensão nos permite ir além no que se refere a mapear o desencadeamento do processo de busca pelo usuário do sistema de informação. Permite visualizar as estratégias adotadas, as dinâmicas de busca envolvidas nas relações entre os conceitos e, principalmente, a capacidade de mobilização de conhecimentos a partir das conexões apresentadas entre os conceitos da rede.

Considerando que o comportamento informacional se configura como uma visível mudança no estado de conhecimento do indivíduo, com o objetivo de utilizar a informação para resolver uma situação específica (BRUM; BARBOSA, 2009), entende-se que tal comportamento é resultado das ações de busca e uso de informação, realizadas por um indivíduo, ou grupo de indivíduos, objetivando atender às suas necessidades de informação (GASQUE; COSTA, 2010).

Nessa perspectiva, e considerando também as definições de Wilson (1997) ao afirmar que o information seeking behaviour é compreendido como o conjunto de métodos à disposição dos usuários para que esses possam acessar fontes de informação e, no caso específico dessa tese, os sistemas de informação, pode-se analisar os métodos de busca de informação e, sobretudo, classificá-los conforme são acionados pelos usuários na busca por informação, sendo possível, inclusive, analisar o nível de interação entre usuários e sistemas de informação a partir dos métodos de busca utilizados.

Em relação ao information search behaviour, Wilson (1997) compreende que essa categoria engloba os mecanismos de interação entre 
usuários e sistemas na busca de informação. Ao tratar especificamente da interação entre usuários de informação e, principalmente, considerar que os usuários interagem com diversos sistemas na busca por informação, percebe-se que essas questões postas por Wilson (1997) estão mapeadas e explicadas a partir de princípios encontrados nos relacionamentos conceituais, como a inexistência de nós desconectados nessa rede - que, por sua vez, informa-nos sobre o inter-relacionamento entre o desencadeamento da busca de informação, os métodos empregados e a interação com as diversas fontes de informação consultadas.

A dinâmica da rede analisada pôde ser verificada ao se constatar que a transitividade entre os métodos empregados pelos usuários dos sistemas de informação e a interação entre eles são facilitadas pela necessidade de poucas conexões entre essas duas categorias. Conforme Guinchat e Menou (1994, p. 481), o usuário é um elemento fundamental de todos os sistemas de informação e, portanto, “[...]é um agente essencial na concepção [...] e funcionamento de qualquer sistema de informação.”. Nessa perspectiva, compreende-se que a ideia de um sistema de informação existe e é justificada pela noção de usuários que os utilizam para atendimento de suas necessidades de informação.

Todo esse conjunto forma um agregado de categorias distintas que se apresentam interligadas de diversas formas e, devido aos agrupamentos em redes menores a partir da identificação desse princípio, pode-se desenvolver estudos que permitam investigar o uso de sistemas de informação desde as mais diversas perspectivas analíticas. Assim, possibilitando conhecer os relacionamentos que se estabelecem entre usuários e os sistemas de informação utilizados para encontrar a informação buscada, bem como os mecanismos estratégicos adotados para encontrar a informação.

Para o campo de conhecimento que trata da Interação, Interfaces, Usabilidade e Mediação da Informação, os princípios levantados a partir da análise do comportamento da rede de temas que o compõe se concentraram na inexistência de nós completamente desconectados e na possibilidade de integração de temas para realização de pesquisas multidisciplinares; por tratar-se de uma rede muito grande em termos lineares, há a dificuldade de se cobrir os pontos mais distantes da rede diretamente; apesar da dimensão da rede, ela 
apresenta baixa densidade de conexões, baixa resistência à retirada de nós aleatoriamente, baixa resiliência, comprometendo a integridade dos diversos componentes que formam a rede, e possibilidade de análises locais ou globais a partir da tendência à formação de comunidades de temas.

A segunda análise verificou questões relativas ao campo da interação humano computador, ou seja, entre usuários e tecnologias de informação em geral, mais diretamente nas relações entre pessoas e tecnologias, quando utilizados para realização de atividades de trabalho ou lazer. Nesse campo de estudos, é possível analisar as questões pertinentes à interação de forma mais aprofundada. Na compreensão de Norman (1986), a interação pode ser compreendida como um processo; nele o usuário apresenta uma necessidade de informação ao formular uma intenção de busca, planejamento das ações necessárias, dialogo em algum nível com a interface do sistema, percepção e interpretação das respostas obtidas no sistema e avaliação acerca do alcance do objetivo inicial (NORMAN, 1986).

Em relação à interface apresentada pelo sistema de informação, essa é o único meio de contato entre o usuário e o sistema (BARBOSA; SILVA, 2010, p. 25); ela estabelece o que o usuário pode fazer, como se comunicar com o sistema e como proceder à realização das tarefas a serem executadas. E, especificamente por oferecerem as dimensões comunicacionais necessárias à execução das atividades que envolvem propriedades das partes que interagem (usuário-sistema) e buscarem manter o equilíbrio entre o poder e o controle exercido pelo usuário (ROCHA; BARANAUSKAS, 2003), a interface deve apresentar ligações com a dimensão de interação. Como visto nas análises nesta seção, essa é uma área muito grande do ponto de vista conceitual e apresenta baixa conectividade entre os termos que compõem a mesma; assim, ainda precisa de desenvolvimentos que fomentem a integração de ações de interação e interface. Com base nos resultados encontrados, é possível verificar que, em ambos os campos, há conhecimentos disponíveis para análises aprofundadas. Entretanto, é necessário buscar maior integração entre elas, o que pode resultar em benefícios para os usuários desses sistemas de informação.

Os princípios levantados a partir da análise do campo temático sobre Tecnologias, Sistemas, Avaliação e Recuperação da Informação concentraram- 
se em determinadas características semelhantes aos princípios levantados nas outras áreas temáticas. Objetivamente podemos dizer que, em cada medida analisada, os princípios foram: inexistência de nós totalmente desconectados e a possibilidade de analisar a mobilidade na rede através do grau de conectividade da mesma; rede muito grande e dificuldade de se cobrir os nós mais distantes; baixa densidade, caracterizando o que pode ser chamado de grafo esparso; baixa resistência à ataques, baixa robustez da rede; e tendência à formação de comunidades de temas em redes menores, possibilitando análises locais e globais da rede.

A discussão relativa ao campo de estudos sobre Tecnologias, Sistemas, Avaliação e Recuperação da Informação, quando analisado do ponto de vista dos estudos de redes, traz uma perspectiva integrada para a compreensão dessas áreas de estudos. A aplicação de medidas de redes permitiu alcançar resultados que mostram a amplitude e a complexidade das áreas de estudo que tratam das tecnologias de comunicação e informação e a recuperação de informação com o uso de tais tecnologias. Especificamente, os resultados mostraram que, individualmente, essas áreas são grandes em relação ao número de conceitos que abarcam; entretanto, a densidade dos relacionamentos entre esses conceitos é muito baixa. Essa situação provoca efeitos nos usos que as pessoas fazem dessas tecnologias, pois, segundo Lévy (1993), elas atuam como agentes externos de processos controlados, os quais requerem o uso contínuo de memória humana de curto prazo.

Esses efeitos são mais visíveis nos processos de recuperação da informação. Esses processos representam um conjunto de atividades realizadas por todos aqueles que buscam informação para atendimento de necessidades informacionais. Além disso, um sistema de informação, em larga medida, pode ser aceito como um conjunto estruturado de elementos ou funções que, em algum nível, interagem entre si, buscando a realização de diversos objetivos. Pode-se, portanto, afirmar que os sistemas de informação influenciam, a partir de cada um de seus componentes, as atividades que os usuários realizam, no sentido de gerar resultados positivos em consonância com os objetivos a serem alcançados. 
Por fim, deve ser salientado que os resultados apresentados nesta seção estão vinculados à estrutura do corpus teórico utilizado. Possivelmente, ao se buscar outro conjunto teórico para interpretar os dados levantados, os resultados obtidos poderão se apresentar sensivelmente diferentes daqueles obtidos nesta tese. Contudo, é necessário ressalvar que o corpus utilizado é exatamente aquele que lida diretamente com as questões postas nesta pesquisa, não fazendo sentido imediato uma hipótese sobre a possibilidade de haver outros resultados a partir do uso de outro corpus teórico, situação que, de partida, seria óbvia, porém interessante.

\section{Considerações finais}

Os resultados alcançados permitiram identificar princípios a partir do comportamento de redes de termos oriundos das áreas de conhecimento delimitadas para a pesquisa. Com o uso de medidas de análise próprias dos estudos de redes, foi possível estruturar um arcabouço metodológico coeso para analisar, de forma reticular, como se apresentam as dinâmicas envolvidas no uso de sistemas de informação que as pessoas fazem diariamente. Cada uma das medidas selecionadas foi aplicada e verificados seus resultados no contexto de três dimensões dos grafos obtidos para cada área de conhecimento. Assim, foram analisadas medidas específicas para a análise de aspectos globais das redes e medidas próprias para análise dos aspectos referentes aos nós da rede. Em todas as medidas analisadas e em todos os grafos elaborados, foi possível identificar ao menos um padrão de comportamento, que ora coincidia com medidas de outras redes, ora divergia em termos comportamentais e topológicos das redes delimitadas na pesquisa.

Cada uma das áreas de conhecimento definidas para a realização da pesquisa apresenta-se como parte integrante de um complexo maior que abarca 3 dimensões distintas, porém complementares. A primeira dessas dimensões refere-se aos fatores humanos presentes nas dinâmicas que compõem o complexo referido acima. Percepção de necessidades humanas que requerem informações específicas para serem sanadas; processos de busca formalizados ou não formalizados, estruturados ou não, desencadeados frente à percepção de 


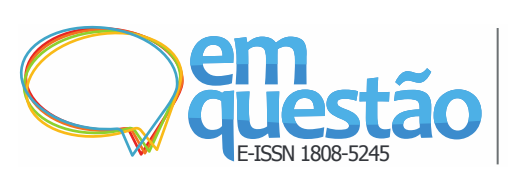

Princípios para análise do uso de sistemas de informação: proposta baseada nos estudos de redes conceituais

Arnaldo Alves Ferreira Junior e Marcelo dos Santos

necessidades de informação; uso sistemático e contínuo de informação como requisito das atividades de trabalho e de lazer do cotidiano. Todas essas questões pertencentes ao componente humano apresentam características particulares que as colocam como uma dimensão específica de análise, diferentes das outras duas dimensões concorrentes.

A segunda área de conhecimento considerada na pesquisa trata da dimensão de interação entre pessoas e sistemas/tecnologias utilizados para manipulação de informação, não levando em consideração os níveis de manipulação ou as finalidades de manipulação dessa informação da qual se fala. Esse posicionamento sobre a dimensão de interação significa que a pesquisa em questão foca nas características dos sistemas/tecnologias de informação voltadas para os relacionamentos que se estabelecem entre homens e máquinas e em que medida se pode considerar tais ferramentas tecnológicas como extensões artificiais da memória de curto prazo dos seres humanos.

Por fim, a terceira área de conhecimento considerada na pesquisa refere-se aos dispositivos tecnológicos de manipulação de informações usados pelas pessoas e as análises que os envolvem, como avaliação desses dispositivos tecnológicos de maneira geral com objetivo de verificar desde a correta implementação do planejamento desses dispositivos até as relações de uso que se estabelecem entre eles e as pessoas que os utilizam, por meio de técnicas de usabilidade. Devido à diversidade e à quantidade desses dispositivos tecnológicos, é necessário compreender a diferença fundamental que há entre o dispositivo em si e o programa que automatiza determinadas funções de manipulação da informação. Nesta pesquisa, foi admitido o termo tecnologias para os dispositivos e sistemas para os programas que fazem tais tecnologias funcionarem.

Ao se utilizar a abordagem de redes para investigar as possibilidades de encontrar novas perspectivas de análise para esses campos de conhecimento, levando-se em consideração que esses campos estão interligados por relacionamentos de diversas naturezas, a expectativa de encontrar princípios de organização e comportamentos específicos a partir do mapeamento de redes de relacionamentos entre esses campos de conhecimentos permeou todo o processo de pesquisa, desde o levantamento de fontes de informação, passando pela 
coleta de dados, organização e tratamento, até a produção dos resultados de pesquisa. A partir dos resultados desse objetivo específico, foram encontrados determinados comportamentos nas redes de relacionamentos, a partir das medidas selecionadas e utilizadas, que puderam ser considerados como os princípios buscados desde o início da pesquisa.

De maneira geral, os princípios encontrados a partir dos comportamentos identificados pela aplicação das medidas de análise de rede indicaram comportamentos semelhantes nas três áreas de conhecimento. Com algumas pequenas divergências em determinadas medidas, explicadas pela especificidade da área de conhecimento, os comportamentos dessas redes modelam os relacionamentos entre áreas de conhecimento voltadas especialmente para os comportamentos humanos na busca de informação, a interação entre os humanos que buscam informação e os dispositivos usados na busca - e também os próprios dispositivos tecnológicos usados.

As redes de relacionamentos de termos identificadas na pesquisa apresentam certas características próprias, as quais foram adotadas como princípios a serem observados quando se pretende avaliar o uso de sistemas de informação. Em cada um desses princípios, foi necessário traçar observações para que se possa interpretar e compreender a dimensão de cada um deles dentro da análise proposta neste trabalho. Ao se buscar referenciais teóricos e, principalmente, metodológicos para pesquisa em Ciência da Informação, originados em áreas distintas de conhecimento, faz-se importante ter a cautela necessária para não fazer uso de determinados conceitos e técnicas das áreas de origem de forma descontextualizada, mas adaptá-las conforme puderem apresentar soluções viáveis a problemas específicos na Ciência da Informação, sem que haja esvaziamento de seus conteúdos essenciais.

Esta pesquisa buscou, em essência, investigar os mecanismos e dinâmicas envolvidos nas situações em que pessoas utilizam sistemas de informação para algum propósito específico, sem necessariamente se preocupar com esses propósitos. Para alcançar as respostas às questões colocadas de início, foram identificadas três áreas de conhecimento que lidam, cada uma conforme suas dinâmicas próprias, com o que foi chamado neste trabalho de usos de sistemas de informação. Ao adotar a estratégia de analisar essa questão a partir da perspectiva 
de integração de áreas de conhecimento, pode-se apreender, de modo estruturado e metodologicamente testado, que problemas complexos podem ser resolvidos com o apoio teórico-metodológico de campos de conhecimento distintos, mas que abarcam em alguma medida os problemas em questão.

Com a conclusão desta pesquisa, podemos afirmar que a investigação dos usos feitos dos sistemas de informação envolve as áreas de comportamentos de busca e uso de informação, a IHC e os sistemas de informação propriamente dito. $\mathrm{O}$ que tornou essa afirmação possível foi o uso da abordagem oriunda dos estudos de redes complexas, que até então contribuía para o campo da Ciência da Informação com estudos sobre redes de citação. Com esta pesquisa, além dos resultados delineados no escopo do projeto, buscou-se aliar os estudos já desenvolvidos com uma nova abordagem metodológica pouco utilizada, os Estudos de Redes Complexas.

\section{Referências}

BARBOSA, S. D. J.; SILVA, B. S. Interação humano computador. Rio de Janeiro: Elsevier, 2010.

BEDERSON, B. B.; SHNEIDERMAN, B. The Craft of Information

Visualization: readings and reflections. San Francisco: Morgan Kaufman, 2003.

BRUM, M. A. C.; BARBOSA, R. R. Comportamento de busca e uso da informação: um estudo com alunos participantes de empresas juniores.

Perspectivas em Ciência da Informação, Belo Horizonte, v. 14, n. 2, p. 52-75, maio/ago. 2009.

CASTELLS, M. A Galáxia da Internet: reflexões sobre a internet, os negócios e a sociedade. Rio de Janeiro: Jorge Zahar, 2003.

CORREIA, P. R. M.; SILVA, A. C. da; ROMANO JUNIOR, J. G. Mapas Conceituais como ferramentas de Avaliação na Sala de Aula. Revista Brasileira de Ensino de Física, v. 32, n. 4, p.1-8, abr. 2010.

GASQUE, K. C. G. D.; COSTA, S. M. de S. Evolução teórico-metodológica dos estudos de comportamento informacional de usuários. Ciência da Informação, Brasília, v. 39, n. 1, p. 21-31, jan./abr., 2010.

GUINCHAT, C.; MENOU, M. Introdução geral às ciências e técnicas da informação e documentação. 2.ed. Brasília: IBICT, 1994.

LÉVY, P. Tecnologias da Inteligência: o futuro da inteligência na era da informática. Rio de Janeiro: 34, 1993. 
MARTÍNEZ-SILVEIRA, M.; ODDONE, N. Necessidades e comportamento informacional: conceituação e modelos. Ciência da Informação, Brasília, v. 36, n. 1, p. 118-127, maio/ago. 2007.

NORMAN, D. A. User centered systems design. New York: Lawrence Earlbaum Associates, 1986.

ROCHA, H. V.; BARANAUSKAS, M. C. C. Design e Avaliação de Interfaces Humano-Computador. Campinas: Universidade Estadual de Campinas, 2003.

SANTA ROSA, J. G.; MORAES, A. Avaliação e Projetos no Design de Interfaces. Teresópolis: 2AB, 2008.

WILSON, T. D. Information behaviour: an interdisciplinary perspective. Information Processing \& Management, Amsterdam, v. 33, n. 4, p. 551-572, 1997.

\title{
Principles for analysis of the use of Information Systems: a proposal based on studies of conceptual
}

\begin{abstract}
For years, studies and evaluations about the use of products and/or services available in digital environments have gained importance, aiming at products and/or services that offer easy handling and pleasant experiences to the users. It happens, however, that users understand the world differently, and consequently their information needs are also different. Thus, based on this premise, the main objective of this research was to identify and characterize guiding principles for the analysis of the use of information systems in digital environments. The research was conducted based on the studies of networks of conceptual relationships. For that, a conceptual framework was used, and, from the forms and behaviors presented by the conceptual connections, the principles were observed and extracted for the analysis of the use of information systems. This is, therefore, a qualitative research, based on the bibliographic method, survey in electronic discussion forums and, in a complementary way, we used methods, techniques and measures of the networks studies for identification and characterization of the principles for evaluation of information systems. As a result, it is highlighted that the identified principles represent guiding elements for the composition of parameters and creation of procedures that help in the analysis of the uses of information systems in general. It's not about replacing the widely used methods and techniques in the areas of Human Computer Interface (IHC), Usability, Systems Analysis and/or Requirements Engineering; however, these principles represent contributions to the study of the interactions between people and information systems, considering the relationships between the concepts that these people activate and/or mobilize to interpret these contexts of use.
\end{abstract}


Princípios para análise do uso de sistemas de informação: proposta baseada nos estudos de redes conceituais

Arnaldo Alves Ferreira Junior e Marcelo dos Santos

Keywords: Use of information. Behaviors of search and use of information. Information systems. Human-computer interaction. Network studies.

Recebido: 19/10/2018

Aceito: $12 / 02 / 2019$ 